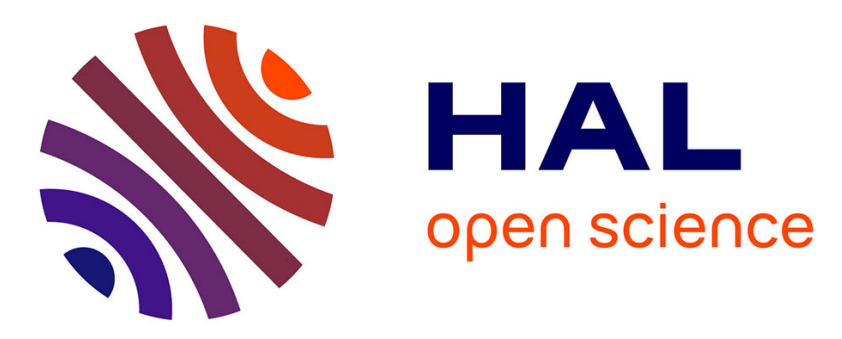

\title{
Le conditionnel dans une langue non tensée: le gbaya de R.CA
}

Paulette Roulon-Doko

\section{To cite this version:}

Paulette Roulon-Doko. Le conditionnel dans une langue non tensée: le gbaya de R.CA. Bres Jacques, Sophie Azzopardi et Sophie Sarrazin. Ultériorité dans le passé, valeurs modales, conditionnel, 40, Peter Lang, pp.181-188, 2013, Faits de Langue, 978-3-0343-1382-7. halshs-01152035

\section{HAL Id: halshs-01152035 https://shs.hal.science/halshs-01152035}

Submitted on 14 May 2015

HAL is a multi-disciplinary open access archive for the deposit and dissemination of scientific research documents, whether they are published or not. The documents may come from teaching and research institutions in France or abroad, or from public or private research centers.
L'archive ouverte pluridisciplinaire HAL, est destinée au dépôt et à la diffusion de documents scientifiques de niveau recherche, publiés ou non, émanant des établissements d'enseignement et de recherche français ou étrangers, des laboratoires publics ou privés. 


\title{
Le conditionnel dans une langue non tensée : le gbaya de R.CA
}

\author{
Paulette Roulon-Doko*
}

En français et dans les langues romanes en général, le conditionnel a une double valeur (i) temporelle qui actualise un procès comme ultérieur à un repère situé dans le passé, et (ii) modale qui prend en compte les conditions de validation. J'étudierai dans une langue non tensée, le gbaya, les procédés verbaux utilisés pour rendre les valeurs du conditionnel en français qui est la langue cible de ce travail. Ayant présenté rapidement les caractéristiques du système verbal gbaya qui s'organise en combinant modes et aspects, je présenterai les emplois du Virtuel accompli, une forme qui prend en charge l'expression de ce qui est irréalisé ou non réalisable. Je montrerai que cette forme ne peut jamais avoir de valeur temporelle mais uniquement des valeurs modales dont j'étudierai les cadres d'emplois. Il s'agit principalement d'un conditionnel d'éventualité. Par ailleurs, je présenterai la seule marque de passé, le Révolu, qui est une modalité de l'élément prédicatif de la phrase, qu'il s'agisse d'un verbe ou d'un prédicat non verbal comme l'Essentiel. C'est un élément qui s'associe de façon remarquable à la présence du Virtuel accompli. Je conclurai sur le statut de ce Virtuel accompli qui n'est pas un temps mais au croisement d'un aspect et d'un mode et exprime toujours l'irréel, un monde différent du monde réel.

\section{LES CARACTERISTIQUES DU SYSTEME VERBAL GBAYA}

Le gbaya est une langue oubanguienne ${ }^{1}$ de type isolante, avec une faible morphologie, sans aucun type d'accord, où l'opposition verbe / nom est bien nette. Le verbe qui exprime toujours un procès ${ }^{2}$ est porteur de marques spécifiques couramment désignées comme TAM (Temps-Aspect-Mode) qui de fait, en gbaya, ne combinent que des modes et des aspects. L'expression du temps est prise en charge par d'autres éléments de la langue (Noms, Adverbes, Phrases "quand le jour se lève" par ex.) qui n'ont pas d'incidence sur les formes

\footnotetext{
*LLACAN, PRESS Paris-Cité, CNRS, INALCO. Courriel : roulon@vjf.cnrs.fr

${ }^{1}$ Le gbaya appartient, selon la classification de Greenberg, au groupe 1 de la branche orientale de la sous-famille 6 "Adamawa oriental" de la famille Niger-Congo.

${ }^{2}$ La prédication non processive est exprimée en gbaya par des prédicatifs non verbaux.
} 
verbales, mais sont nécessaires si l'on veut situer l'événement dans un temps précis. Par ailleurs, le temps relatif est déductible de l'agencement dans une succession des diverses formes verbales.

L'ensemble des modalités obligatoires forment le paradigme de la conjugaison qui comprend 20 formes organisées selon deux aspects - Inaccompli et Accompli - et trois modes - Réel, Virtuel et Nécessaire. Tout procès peut ainsi être soit exprimé comme un fait : c'est le mode Réel ; soit comme un projet ou une éventualité : c'est le mode Virtuel ; soit comme un ordre : c'est le mode Nécessaire. L'opposition d'aspects INACC / ACC qui intervient au sein de chaque mode n'y manifeste pas la même valeur. Au mode Réel, l'inaccompli marque un procès vu de l'intérieur, c'est-àdire dans sa saisie cursive et l'accompli un procès vu de l'extérieur dont on souligne la globalité comme un tout indivis. Au mode Virtuel, l'inaccompli indique un point de vue prospectif, sans prise en charge de la réalisation possible ou non du procès, et l'accompli une valeur d'irréel signalant que le procès ne peut plus être réalisé. Au mode Nécessaire, l'inaccompli exprime l'ordre qui attend d'être réalisé, qu'il le soit ou non, et l'accompli un ordre à valeur performative, c'est-à-dire considéré comme déjà réalisé. A ces formes conjuguées peuvent venir s'ajouter des marques expansives - Révolu, Habituel, Instrumental, etc. Il y a une stricte distribution de ces deux types de déterminants (Dt) de part et d'autre de la base verbale : $<\mathrm{Dt}$ obligatoires ${ }^{3}+\mathrm{BV}+\mathrm{Dt}$ expansifs $>$.

En gbaya, c'est dans le mode Virtuel où tout procès est exprimé comme un projet ou une éventualité que va se trouver une forme qui peut rendre ce que le français exprime par un conditionnel : le Virtuel accompli (V.ACC). Il s'agit d'une forme périphrastique qui utilise le verbe $t \varepsilon$ "venir" comme auxiliaire. Cet auxiliaire précède la base verbale affectée d'un schème tonal bas, constituant la

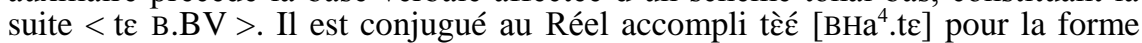
accomplie du Virtuel et au Réel inaccompli té [H.tદ] pour la forme inaccomplie du Virtuel (V.INACC). On peut signaler ici que la forme accomplie de l'auxiliaire ṫ̀é est également utilisée pour marquer les formes accomplies de deux autres formes, en se rajoutant tout simplement à leur forme d'inaccompli. Il n'y a alors pas de parallélisme de construction entre leurs formes ACC / INACC comme c'est le cas pour les deux formes du Virtuel. Ensemble, ces deux formes ont une fréquence d'emploi dans mon corpus de référence d'environ $8 \%$. La forme inaccomplie du Virtuel exprime un possible - projet ou éventualité - souvent rendu dans la traduction française par un futur. Mais contrairement à la valeur de ce dernier en français, il ne porte jamais une valeur de programmation dans le

\footnotetext{
${ }^{3}$ Placés devant la base verbale ou dessus, sous forme tonale, la base verbale n'ayant pas de schème tonal lexical propre.

${ }^{4} \mathrm{Le}$ 'a' marque la forme longue du verbe qui, dans le cas d'un verbe CV comme $t \varepsilon$, est
} réalisée $\mathrm{CVV}$ et non $\mathrm{CVa}$. 
temps ${ }^{5}$. La forme accomplie du Virtuel exprime, elle, une action dont la réalisation est impossible ou posée comme telle, elle a une valeur d'irréel. C'est cette forme qui pourra le plus souvent être traduite en français par un conditionnel présent ou passé, selon le cotexte.

Je présenterai les emplois du Virtuel accompli tout d'abord dans le cadre d'une proposition indépendante puis, lorsqu'il se trouve dans une proposition en apodose, après une proposition conditionnelle.

\section{LE VIRTUEL ACCOMPLI DANS UNE PROPOSITION INDEPENDANTE}

Employé dans une proposition indépendante, le Virtuel accompli prend en charge des valeurs modales comparables pour certaines à celles retenues par Dendale (2001 : 9) pour le conditionnel en français. Toutes ces valeurs ont en commun d'exprimer un procès (projet ou éventualité) dont la réalisation est, au moment de l'énonciation, posée comme irréelle.

\subsection{L'éventualité}

L'éventualité dont on sait qu'elle n'a pu être réalisée est exprimée par le Virtuel accompli. Il peut être traduit en français soit par un conditionnel, soit par "avoir pu" et aussi "avoir dû" au conditionnel.

(1)

\begin{tabular}{|c|c|c|c|}
\hline $\begin{array}{l}\text { kínè } \\
\text { maintenant }\end{array}$ & $\begin{array}{l}\mathrm{m} \varepsilon \\
2 \mathrm{SG}\end{array}$ & $\begin{array}{l}\text { tècé-kpăm } \\
\text { V.ACC.trouver.1 SG à. } \\
\text { à. }\end{array}$ & $\begin{array}{l}\text { sálá } \\
\text { NV écarter }\end{array}$ \\
\hline $\begin{array}{l}\text { maintenant } \\
\text { yù péí-dòn } \\
\text { fuite en_arrière }\end{array}$ & $\begin{array}{l}2 \mathrm{SG} \\
\text { hغ્. } \\
\text { ici }\end{array}$ & & \\
\hline
\end{tabular}

"Tout de suite ici, tu m'aurais trouvé en train de revenir en courant jusqu'ici."

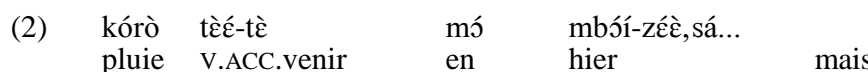

"Hier il aurait bien plu, mais ${ }^{7}$...." [le vent à chassé les nuages]

(3) Pá ndàá fiò nè wàntò tèć-fề

voilà que raison.D mort REL Npr V.ACC.mourir

yè, mè né náná rìkìsí

en effet chose ETRE-ESS marche.D fourberie

${ }^{5}$ Le futur programmable est rendu par un Réel accompli précédé d'une marque lexicale de futur (demain, bientôt, dans deux jours...).

${ }^{6}$ Les gloses correspondent aux abréviations recommandées par Faits de Langues auxquelles il faut ajouter POL "forme de politesse", D "relateur tonal", INJ "injonctif" et des abréviations qui sont présentés dans le texte de l'article comme v "virtuel" par exemple.

${ }^{7}$ Le développement qui suit n'est pas une condition mais une explication qui pourrait ne pas être dite sans changer la valeur de la première proposition. 
$\begin{array}{llll}\text { Pá-nè } & \text { wàntò } & \text { tèć-fè } & \text { sèné } \\ \text { TOP } & \text { Npr } & \text { V.ACC.mourir } & \text { dedans }\end{array}$

"Et la cause de la mort qui aurait pu en effet tuer Wanto, c'est sa manie de ruser qui aurait pu tuer Wanto."

(4) Péné tèć-Tànì só nàà mó Pẽ

2POL V.ACC.demander REV mère à savoir celle-ci d'abord

"Vous auriez pu d'abord questionner cette mère-ci." (mais vous ne l'avez pas fait)

Dans cet exemple, la présence du Révolu só qui marque un passé non spécifié comme je le présenterai ci-après, ancre la valeur d'irréel du V.ACC dans le passé.

\subsection{L'emprunt}

Cette valeur médiative du V.ACC signale une information empruntée qui consiste à reprendre les paroles d'autrui en marquant une distance.

$\begin{array}{llll}\text { (5) Tám } & \text { tèć-bà } & \text { lààí hógá } & \\ & \text { 1SG } & \text { V.ACC.prendre } & \text { linge.ANAPH } \\ & \text { comme_ça }\end{array}$

"J'aurais ainsi pris ce tissu...." [Voilà ce qu'il a dit pour me faire honte]

\subsection{Le regret ou le souhait}

Le V.ACC a ici une valeur illocutoire, le locuteur manifestant regret ou souhait.

Pám tèć-kpà kàà wí Pèm néné !

1SG V.ACC.trouver aussi.D homme.D à.accompagner.1SG en allant

"Ah! Si je trouvais quelqu'un pour m'accompagner !" (litt. Je trouverais quelqu'un pour m'accompagner !)

\section{LE VIRTUEL ACCOMPLI DANS UNE PROPOSITION CONDITIONNELLE}

Le plus souvent, c'est au sein d'une proposition conditionnelle, associant antécédent et conséquent qu'on trouve en apodose un verbe au Virtuel accompli. Je parlerai ici d'un conditionnel d'éventualité spécifié selon la définition de Dendale (2001 : 11) comme un "emploi d'éventualité en période hypothétique (avec une conditionnelle se rattachant au contenu propositionnel de la principale)". En gbaya, deux cas se présentent, selon que l'antécédent est (i) une conditionnelle contrefactuelle ou (ii) une conditionnelle simple. J'examinerai successivement les deux cas. 


\subsection{Une conditionnelle contrefactuelle}

En gbaya, la conditionnelle contrefactuelle est repérée par la présence du Révolu qui va situer l'antécédent (P1) dans le passé, identifiant ainsi une sphère de temps reculé qui n'est plus d'actualité, sans qu'un moment précis ne soit spécifié.

L'aspectif $s \delta ́$ appelé 'Révolu' et noté REV dans le mot à mot, est un terme original qui indique toujours une mise en relation avec le passé. Il s'agit d'une modalité de prédication qui porte aussi bien sur le verbe - en tant qu'expansion facultative ${ }^{8}-$ que sur les prédicatifs non verbaux. Dans une proposition verbale indépendante, l'adjonction du Révolu signale dans tous les cas une valeur de passé qui va exprimer des degrés différents, selon la forme conjuguée du verbe.

Avec un Réel inaccompli pour la forme conjuguée (procès générique ou qui se développe) le Révolu signale un procès en développement rendu le plus souvent en français par un imparfait comme dans l'exemple suivant où il s'agit d'une suite de lamentations de l'épouse dont le mari est mort.

(8) wéì kóm gbé só sàdî wó mari de.1SG INACC.tuer ${ }^{2}$ REV vraiment "Mon mari qui tuait du gibier!"

Avec un Réel accompli pour la forme conjuguée (procès global) le Révolu signale un événement qui a bien eu lieu. Il peut être rendu en français par un plus-que-parfait comme dans l'exemple suivant où le locuteur rappelle l'enjeu du pari que l'autre a perdu.

$\begin{array}{lllll}\text { mé } & \text { tèc̀ } & \text { s.́ } & \text { bá } & \text { kóòí sèn } \\ \text { 2SG } & \text { ACC.venir.D } & \text { REV } & \text { I.ACC.prendre } & \text { épouse.ANAPH } \\ \text { sèn } & \text { PÉrám } & \text { ndé } & & \\ \text { dans } & \text { main.D.1SG } & \text { DISC } & & \\ \text { "Tu étais venu prendre une épouse chez moi." } & \end{array}$

Dans le cas d'une succession de deux propositions qui comporte en protase un Révolu et en apodose un V.ACC, le Révolu présent en P1 est toujours associé à un verbe conjugué au Réel inaccompli-noté INACC dans le mot à mot. On a la succession: <P1 [INACC + Révolu] / P2 [V.ACC] >. Dans cette construction, P1 acquiert, en l'absence de toute marque de conjonction, un statut de conditionnelle contrefactuelle représentant l'antécédent dont la principale P2 est le conséquent. Le verbe au V.ACC du conséquent (P2) est rendu en français par un conditionnel passé ou présent selon le cotexte. On saisit ici l'importance de l'agencement des propositions puisque si la protase était dite seule cela nous conduirait à une valeur d'imparfait "tu aimais Naa-ngo'di" comme dans l'ex. 8.

${ }^{8}$ C'est pour le verbe une expansion qui ne participe jamais à la conjugaison (formes obligatoires) mais s'y ajoute. 

(10) mé k2́ só nàá-ngòdí wó

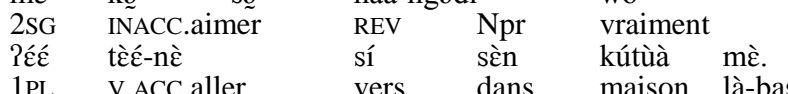
"Si tu aimais Naa-ngo'di, nous irions dans la maison là-bas."
(11) Rám Rín só Rám tèce-nè gíà
1SG INACC.savoir REV 1SG V.ACC.aller chasse
kóm Tèá wó.
de.1SG seulement vraiment
"Si j'avais su, assurément je serais seulement allé à la chasse."
(12)

\begin{tabular}{|c|c|c|c|c|}
\hline ?á & kà & bé & só & né \\
\hline $\begin{array}{l}\text { c'est } \\
\text { ná }\end{array}$ & $\begin{array}{l}\text { SUB.alors.D } \\
\text { wó }\end{array}$ & INACC.pouvoir_être & $\begin{array}{l}\text { REV } \\
\text { béí }\end{array}$ & $\begin{array}{l}\text { ETRE-ESS 3SG } \\
\text { tèć-kàì }\end{array}$ \\
\hline $\begin{array}{l}\text { NEG } \\
\text { kóò }\end{array}$ & $\begin{array}{l}\text { vraiment } \\
\text { yíitòó }\end{array}$ & $\begin{array}{l}\text { INACC.pouvoir_être.INS } \\
\text { ná. }\end{array}$ & gens & V.ACC.pr \\
\hline
\end{tabular}
kóo yíítòó ná.
épouse deux NEG épouses."

Le V.ACC du conséquent est dans tous ces exemples une action qui exprime un irréel renvoyant à un monde différent du monde Réel.

\subsection{Une conditionnelle simple}

La conditionnelle simple est repérée en gbaya par la seule présence dans l'antécédent (P1) d'une forme du Réel appelée "hypothétique ${ }^{10 "}$ et notée HYPO dans le mot à mot. Sur le plan formel, le Réel hypothétique est une forme composée comportant l'essentiel né ETRE-ESS, un prédicatif non verbal qui précède la base verbale conjuguée au Réel accompli, posant donc l'hypothèse comme un fait validant la globalité du procès qu'elle mentionne. Le plus souvent, la proposition principale qui suit en apodose, le conséquent, a un verbe soit à l'accompli du Réel, soit à l'injonctif. Dans tous ces cas, l'hypothèse ainsi exprimée définit le cadre où pourra se situer l'action du verbe en $\mathrm{P} 2$ dont le procès reste réalisable. Ce cadre servant ainsi de référence temporelle, l'apodose ne peut plus alors accepter un verbe au Virtuel inaccompli qui, par définition, signale l'action comme un projet dans un avenir non spécifié et ne permet pas de le programmer contrairement aux autres formes qui viennent d'être mentionnées. En français, la

\footnotetext{
${ }^{9}$ La présence du verbe potentiel permet de faire porter la négation sur un élément autre que le verbe qui suit, ici le terme "deux". Ils prendraient une épouse mais pas deux. L'analyse du rôle de ce verbe déborde le cadre de cet exposé.

${ }^{10}$ Il s'agit d'une forme liée, c'est-à-dire une forme qui par sa seule présence intègre la proposition qui la contient dans une structure hiérarchique dont elle est, dans le cas de l'hypothétique, la protase.
} 
conditionnelle simple sera rendue par "si + présent" ou "quand + futur". C'est toujours le cotexte qui donne la position sur l'axe du temps.

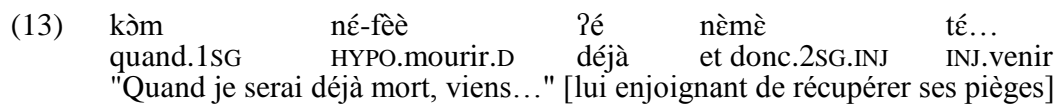

Par contre, lorsque le verbe en $\mathrm{P} 2$ est au V.ACC cela signale que le procès envisagé n'a pu se réaliser et n'est plus réalisable, inférant que la condition spécifiée n'est plus d'actualité bien qu'aucune marque explicite ne renvoie au passé comme pouvait le faire le Révolu dans les exemples précédents. Ce verbe au V.ACC est en français traduit par un conditionnel comme dans l'ex.12. S'agissant d'un discours produit lors d'un jugement qui relate des faits déjà anciens, en français, on emploiera nécessairement des temps du passé et, en respectant la concordance des temps, des conditionnels à valeur temporelle parallèles à des futurs dans un discours au présent.

[Kàá-ndì̀ était allée, quant à elle, faire sécher du manioc. Le soleil n'avait pas brillé et Kàá-ndì̀ avait pris le chemin du retour.]

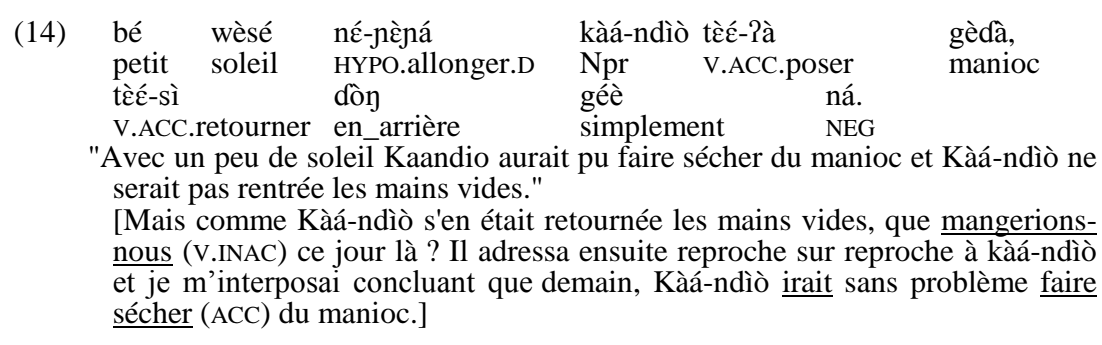

Les deux conditionnels marqués par un soulignement dans la traduction française, expriment la valeur temporelle du Conditionnel marquant l'ultériorité dans le passé. En gbaya, le premier est exprimé par un Virtuel inaccompli soulignant que le projet reste réalisable (ils ont bien sûr mangé quelque chose) et le second par un Réel accompli qui est la façon, lorsqu'un espace temporel est ouvert - demain - de mentionner le procès programmé pour s'y réaliser. Les V.ACC de l'ex.12 expriment, eux, des procès qui resteront irréels : il s'agit d'une valeur modale d'éventualité et aucunement d'une valeur temporelle.

Ainsi dans le cas peu fréquent d'une conditionnelle simple, la seule présence d'un verbe au V.ACC en apodose suffit à marquer la valeur d'irréel, sans prendre en charge une ultériorité dans le passé. On est renvoyé dans un monde différent du monde réel, comme c'était le cas pour les hypothétiques contrefactuelles.

\subsection{Révolu et Essentiel}


Lorsque le Révolu porte, au sein de phrases interrogatives, sur le prédicatif

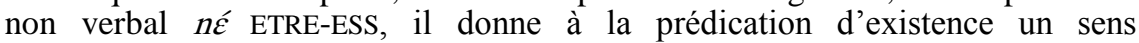
conditionnel que je rends littéralement par "se pourrait-il ?". Cette construction, très courante pour exprimer l'étonnement, peut être appelée conditionnel d'hypothèse (cf. Dendale, $2004: 8$ ).

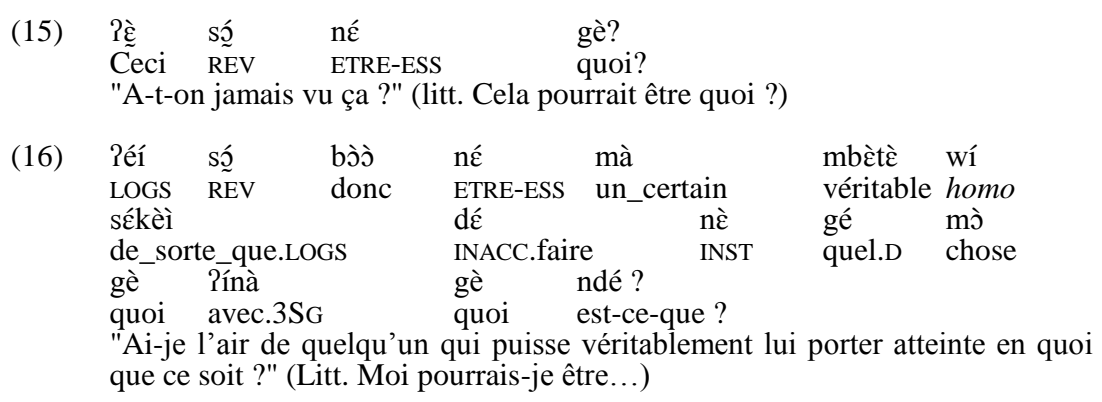

\section{CONCLUSION}

En gbaya, la prise en compte d'une valeur générique de passé relève d'une modalité, le Révolu, qui est toujours une expansion pour le syntagme verbal. Sa présence qui est fondamentale pour repérer une conditionnelle contrefactuelle permet aussi, en se portant sur l'Essentiel, l'expression d'un conditionnel d'hypothèse, réalisé sans support verbal. Au sein du paradigme des formes verbales conjuguées (modalités obligatoires et mutuellement exclusives) le Virtuel accompli n'est ni un mode ni un temps, mais une forme verbale qui combine mode Virtuel (projet, éventualité) et aspect Accompli (globalité du procès) et dont la valeur unique exprime dans tous ses emplois un procès dont la réalisation n'a pu se faire et n'est plus réalisable. Son invariant sémantique est sa valeur d'irréel qui renvoie à un monde différent du monde réel et ne réfère à aucun temps particulier, mais manifeste des valeurs uniquement modales relevant de façon majoritaire de l'éventualité.

\section{REFERENCES BIBLIOGRAPHIQUES}

Dendale P., 2001, Les problèmes linguistiques du conditionnel francais, in P. Dendale et L. Tamowski, (éds), Le conditionnel en français, Mets, Université de Mets / Paris, Klinksieck, Coll. Recherches Linguistiques 25, p. 7-18.

Moeschler J. et A. Reboul, 2001, Conditionnel et assertions conditionnelles, in P. Dendale \& L. Tasmowski (éds), Le conditionnel (en français), Paris, Klinsieck, p. 147-167. 
Tournadre N., 2004, Typologie des aspects verbaux et intégration à une théorie du TAM, $B S L$ XCIX, fasc. 1, p. 7-68. 